\title{
CONCERNING ZERO-DIMENSIONAL SETS IN EUCLIDEAN SPACE*
}

\author{
BY
}

R. L. WILDER

A point set $M$, lying in a euclidean space, $E_{n}$, of $n$ dimensions, is said to be zero-dimensional in the Menger-Urysohn sense, if for every point $P$ of $M$ and every positive number $\epsilon$ there exists a separation of $M$ into two mutually separated sets $M_{1}$ and $M_{2}$ such that $M_{1}$ contains $P$ and the diameter of $M_{1}$ is less than $\epsilon$. $\dagger$

The present paper is intended to serve as a contribution to the study of zero-dimensional sets in $E_{n}$, particularly with reference to the relations, of these sets to their complements. The property of accessibility of a point set from all sides is introduced and it is shown that in $E_{n}(n>1)$ all zero-dimensional sets possess this property and in $E_{2}$ are characterized by it. An example is given to show that, in the definition of accessibility used, arcs cannot be employed instead of continua. The same example shows that if $M$ is a zero-dimensional set in $E_{2}$, then, although it is well known that $M$ is homeomorphic with a subset of $I_{2}$, the set of all points in $E_{2}$ both of whose coördinates are irrational, there does not in general exist a one-to-one continuous transformation of $E_{2}$ into itself which carries $M$ into a subset of $I_{2}$; i.e., $M$ is not in general isotopic with a subset of $I_{2}$. A necessary and sufficient condition is then obtained under which $M$ will be isotopic with a subset of $I_{2}$, and by application of this condition it is found that every punctiform $F_{\sigma}$ in $E_{2}$ is isotopic with a subset of $I_{2}$.

DeFINITION. In this paper, the term region will be used to denote a simply connected, bounded domain.

It follows from a theorem of Sierpinskif that zero-dimensional sets are

* Presented to the Society, April 15, 1927, under the title Concerning zero-dimensional sets in the plane; received by the editors March 31, 1928.

$\dagger$ For a general summary of the Menger-Urysohn dimension theory and references, see $K$. Menger, Bericht über die Dimensionstheorie, Jahresbericht der Deutschen Mathematiker-Vereinigung, vol. 35 (1926), pp. 113-150.

$\ddagger$ W. Sierpinski, Sur les ensembles connexes et non connexes, Fundamenta Mathematicae, vol. 2 (1921), pp. 81-95. 
identical with those sets that are punctiform and homeomorphic* with linear sets. Mazurkiewicz has shown $\dagger$ that if $a_{1}$ and $a_{2}$ are points of a punctiform set $A$ in $E_{n}$ which is homeomorphic with a linear set, and $D$ is a domain of $E_{n}$ containing $a_{1}$, then there exists a continuum $C$ which lies wholly in $D$, contains no point of $A$ and separates $a_{1}$ from $a_{2}$. Hence, if $M$ is a zerodimensional set in $E_{2}, P$ and $Q$ are distinct points of $M, \epsilon$ is any positive number less than the distance from $P$ to $Q$, and $D$ is the set of all points whose distance from $P$ is less than $\epsilon$, there exists a set $F$ which is a subcontinuum of $D-D \times M$ and which separates $P$ from $Q$. If $R$ is that component $\ddagger$ of $E_{2}-F$ determined by $P$, then $R$ is a domain. Its boundary, $B$, is a continuum by virtue of a theorem of Brouwer; $\S$ hence $R$ is simply connected\| and consequently a region as defined above, its boundedness being evident. That $B$ contains no point of $M$ is obvious, since $B$ is a subset of $F$.

If a point set $M$ in $E_{2}$ has the property that for every point $P$ of $M$ and every positive number $\epsilon$ there exists a region containing $P$ every point of which is at a distance from $P$ less than $\epsilon$ and whose boundary contains no point of $M$, then it is obvious that $M$ is zero-dimensional. We have then the following lemma:

LEMMA 1. In order that a point set $M$ in $E_{2}$ should be zero-dimensional it is necessary and sufficient that for every point $P$ of $M$ and every positive number $\epsilon$ there exist a region containing $P$ every point of which is at a distance from $P$ less than $\epsilon$ and whose boundary contains no point of $M$.

Definition. If $P$ is a point of a point set $M$ in $E_{2}$, then $M$ will be said to be locally separated at $P$ provided that for every positive number $\epsilon$ there exists a region containing $P$ whose diameter is less than $\epsilon$ and whose boundary contains no point of $M$. If $M$ is locally separated at all of its points, then $M$ will be called locally separated.

* Two sets $M$ and $N$ are said to be homeomorphic if there exists a one-to-one continuous correspondence between them. A set is called punctiform if it contains no continuum. (A continuum is a closed and connected point set containing more than one point.)

$\dagger$ S. Mazurkiewicz, Sur un ensemble $G_{\delta}$, punctiforme, qui n'est pas homeomorphe avec aucun ensemble linéaire, Fundamenta Mathematicae, vol. 1 (1920), pp. 61-81, Theorem IV. It is clearly intended that the word "punctiforme" appear in this theorem-thus: " $A$ est un ensemble punctiforme de $R_{q} \cdots$. ."

If $M$ is a point set and $P$ a point of $M$, then that component of $M$ determined by $P$ is the set of all points $\{x\}$, of $M$, such that $x$ and $P$ lie in a connected subset of $M$.

$\S$ Cf. L. E. J. Brouwer, Beweis des Jordanschen Kurvensatzes, Mathematische Annalen, vol. 69 (1910), pp. 169-175, Theorem 3.

II Cf. R. L. Moore, Concerning continuous curves in the plane, Mathematische Zeitschrift, vol. 15 (1922), pp. 254-260, Theorem 2. 
Definition. A sequence of regions, $G$, is said to close down on a point $P$ if every region of $G$ contains $P$ and if for every positive number $\epsilon$ all but a finite number of regions of $G$ are of diameter less than $\epsilon$.

Definition. A set of regions, $G$, is said to cover a point set $M$ in the $V$ itali sense provided that if $P$ is any point of $M$ there exists an infinite sequence of regions of $G$ closing down on $P$.

Definition. A set of regions, $G$, is said to have property $\mathrm{H}$ if for every positive number $\epsilon$ there exist only a finite number of regions of $G$ of diameter greater than $\epsilon$.

Definition. A point $P$ of a set $M$ in $E_{2}$ will be said to be accessible from all sides provided that if $D$ is a Jordan region* whose boundary contains $P$, then $P$ can be joined to any point $x$ of $D$ by a continuum $C$ which lies wholly in $D$ except for $P$, and such that all points of $C$ except $P$ and possibly $x$ are points of $E_{2}-M$. If in this definition the words "a continuum" are replaced by "an arc," the point $P$ will be called arcwise accessible from all sides.

Definition. If $M$ is a set of points in $E_{2}$, then $E_{2}$ will be said to be accessible from all sides with respect to $M$ if every point $P$ of $E_{2}$ is accessible from all sides when $P$ is added to $M$. An analogous definition for "arcwise accessible from all sides with respect to $M$ " is obvious.

Definition. Two sets $M$ and $N$ lying in spaces $S$ and $T$, respectively, are said to be isotopic in case there exists a one-to-one continuous correspondence between $S$ and $T$ under which $M$ and $N$ correspond to one another. Obviously $S$ and $T$ can be the same space.

Definition. If $P$ is a point of a point set $M$ in $E_{2}$, then $M$ will be said to be simply locally separated at $P$ provided that for every positive number $\epsilon$ there exists a region containing $P$ whose diameter is less than $\epsilon$ and whose boundary is a simple closed curve which contains no point of $M$. If $M$ is simply locally separated at all of its points, then $M$ will itself be called simply locally separated.

Lemma 2. If $M$ is a zero-dimensional set, and $P$ is a point not belonging to $M$, then $M+P$ is zero-dimensional.

This lemma is an immediate consequence of a result due to Urysohn, $†$ to the effect that the set of points at which a set $T$ is of dimension $\geqq 0$ is dense in itself.

* A Jordan region is a bounded domain complementary to a simple closed curve.

$\dagger$ P. Urysohn, Sur les multiplicités Cantoriennes, Fundamenta Mathematicae, vol. 7 (1925), pp. 30-137, and vol. 8 (1926), pp. 225-359. See vol. 8, pp. 272-273. 
THEOREM 1. If $M$ is a zero-dimensional set in $E_{2}$ there exists a sequence of regions $G$ whose boundaries contain no points of $M$, which cover $M$ in the $V$ itali sense, and such that $G$ has property $\mathrm{H} . *$

Consider first a bounded zero-dimensional set $M$. Let $M^{\prime}$ denote the set composed of $M$ together with all its limit points. By Lemmas 1 and 2, if $P$ is any point of $M^{\prime}$, there exists a sequence of regions, $G(P)$, closing down on $P$ whose boundaries contain no point of $M$. Let $G^{\prime}$ be the collection of all regions belonging to sequences of the type $G(P)$. By the Borel Theorem there exists a finite set, $G_{1}$, of regions of $G^{\prime}$ covering $M^{\prime}$. From $G^{\prime}$ omit all regions of diameter $>1$ and call the resulting set of regions $G_{1}^{\prime}$. As $G_{1}^{\prime}$ covers $M^{\prime}$, there exists a finite subset, $G_{2}$, of $G_{1}^{\prime}$, which covers $M^{\prime}$. From $G_{1}^{\prime}$ omit all regions of diameter $>\frac{1}{2}$ and call the resulting set of regions $G_{2}^{\prime}$. In general, if $G_{n}^{\prime}$ consists of the set of all regions of $G^{\prime}$ of diameter $\leqq 1 / n$, there exists a finite subset, $G_{n+1}$, of $G_{n}{ }^{\prime}$, which covers $M^{\prime}$.

Let $G=\sum_{1}^{\infty} G_{n}$. Then if $\epsilon$ is any positive number, only a finite number of regions of $G$ are of diameter $>\epsilon$, and furthermore, since for every point $P$ of $M$ there exists a region of $G_{n}(n=1,2,3, \cdots)$ covering $P, G$ covers $M$ in the Vitali sense.

Since every unbounded set in $E_{2}$ is the sum of a denumerable collection of bounded sets, the set $M$, if unbounded, is the sum of a sequence of bounded sets $M_{1}, M_{2}, M_{3}, \cdots$. The set $G^{\prime}$ can be selected as before; in general $G_{n}^{\prime}$ can consist of all regions of $G^{\prime}$ of diameter $\leqq 1 / n$ and $G_{n+1}$ can be a finite set of regions of $G_{n}^{\prime}$ covering the set $\sum_{1}^{n} M_{i}$ together with its limit points.

THEOREM 1a. If $M$ is a simply locally separated set in $E_{2}$, there exists a set of Jordan regions $G$ whose boundaries contain no point of $M$, which cover $M$ in the Vitali sense, and such that $G$ has property $\mathrm{H}$.

THEOREM 2. If $M$ is a zero-dimensional set in $E_{2}$, then $E_{2}$ is accessible from all sides with respect to $M$.

Let $D$ be a Jordan region and $P$ a point on the boundary, $B$, of $D$. Let $Q$ be any point of $D$. There exists a simple closed curve $J$ which contains $P$ and $Q$ and which lies, except for $P$, wholly in $D$. The curve $J$ is the sum of two arcs, $t_{1}$ and $t_{2}$, which have in common only the points $P$ and $Q$.

Let $z$ be a point of $R$, the region bounded by $J$, and let $t$ be an arc whose end points are $P$ and $Q$, which contains $z$, and which lies, except for $P$ and $Q$, wholly in $R$. Let $M_{1}$ be the set of points common to $M$ and $J-(P+Q)$.

\footnotetext{
* Note added in proof-reading: Since I have recently shown (in my paper Concerning the Phragmen-Brouwer Theorem, presented to the American Mathematical Society, December 27, 1928) 1 and consequently Theorem 1 are true in $E_{n}$, for $n>2$.
} 
As $M$ is zero-dimensional, there exists, by Theorem 1 , a set of regions $G$ which covers $M$ in the Vitali sense and has property $\mathrm{H}$, and such that the boundaries of regions of $G$ contain no points of $M$.

If $x$ is any point of $M_{1}$, there exists a region of $G, g(x)$, which contains $x$ and such that $g(x)$ together with its boundary lies wholly in $D$ and contains no point of $t$. The set of all points contained in regions of the type $g(x)$, together with their boundaries, denote by $T$. That the set of points $T+J$ is a continuum $C$ is easily shown from the properties of $G$.

Let that connected domain complementary to $C$, which contains $z$, be denoted by $D(z)$. The boundary, $F$, of $D(z)$ is a subset of $C$ and that it contains $P$ and $Q$ is easily seen from the fact that all of $t$, except $P$ and $Q$, lies in $D(z)$. That $F$ is a continuum follows from the Brouwer theorem referred to above, and that it contains no point of $M$ except possibly $P$ and $Q$ is obvious. Hence $F$ is a continuum which contains $P$ and $Q$, contains no point of $M$ except possibly $P$ and $Q$, and lies, except for $P$, wholly in $D$.

Theorem 3. If $M$ is a zero-dimensional set in $E_{2}$ and $P$ and $Q$ are any two points of $E_{2}$, then $P$ and $Q$ can be joined by a continuum $K$ every point of which, except possibly $P$ and $Q$, is in $E_{2}-M$; and indeed, if $J$ is any simple closed curve enclosing both $P$ and $Q, K$ may be selected so as to lie entirely within $J$.

From the above it is evident that whereas, by a theorem of Sierpinski,* the complement of a punctiform set in $E_{2}$ is connected im kleinen, $\dagger$ the complement of a set having the stronger property of zero-dimensionality is strongly connected im kleinen.

If, when considering space $E_{n}(n>2)$, we define accessibility from all sides as above, except that Jordan regions are replaced by bounded domains complementary to $n$-dimensional spheres, the above results are easily extended to higher spaces. Thus, we have the following theorem:

Theorem 4. If $M$ is a zero-dimensional set in $E_{n}(n>1)$ then all points of $E_{n}$ are accessible from all sides with respect to $M$, and the complement, $E_{n}-M$, is strongly connected im kleinen.

I shall merely indicate how the proof is given for $E_{3}$. Let $S$ be a sphere, $P$

*W. Sierpinski, Sur un ensemble punctiforme connexe, Fundamenta Mathematicae, vol. 1 (1920), pp. 7-10.

$\dagger$ A set $M$ is called connected im kleinen provided that if $P$ is any point of $M$ and $\epsilon$ is any positive number, there exists a positive number $\rho$ such that if $Q$ is a point at a distance from $P$ less $\operatorname{than} \rho$, there exists a connected subset $N$ of $M$ containing both $P$ and $Q$ every point of which is at a distance from $P$ less than $\epsilon$. If $N$ can always be taken to be a continuum, then $M$ is called strongly connected im kleinen. 
a point on $S$ and $Q$ a point within $S$. Let $T$ be any plane passing through $P$ and $Q$. The intersection of $S$ with $T$ is a circle $C$, and with $M$ is a point set $m$. The set $m$ is zero-dimensional, and by Theorem 3 there exists a continuum $K$ lying entirely within $C$, on $T$, and containing $P$ and $Q$ but no points of $m$ except possibly $P$ and $Q$. The rest of the proof should be obvious.

THEOREM 4a. If, in $E_{n}(n>1), D$ is a connected domain and $M$ is a zerodimensional set and $P$ and $Q$ are distinct points of $D$, then there exists, in $D$, a continuum $C$ which contains $P$ and $Q$ but which contains no point of $M$, except possibly $P$ and $Q$.

I shall indicate the proof for $E_{3}$. (For $E_{2}$ use Theorem 3 and the notion of simple chain indicated below.) Every point $x$ of $D$ is the center of a sphere $S_{x}$ which lies wholly in $D$. Let $G$ denote the collection of all such spheres. Then there exists, from $P$ to $Q$, a simple chain, $S_{1}, S_{2}, \cdots, S_{k}$, of spheres* of the collection $G$. For each $i(i=1,2, \cdots, k-1)$ let $P_{i}$ denote a point of $E_{3}-M$ common to $S_{i}$ and $S_{i+1}$. By passing a plane through $P_{i}$ and $P_{i+1}$ and proceeding as in the proof of Theorem 4 , it can be shown that there exists a continuum $C_{i+1}$ which lies wholly in $S_{i+1}$, contains $P_{i}$ and $P_{i+1}$, but no point of $M$. Similar continua $C_{1}$ and $C_{k}$ can be obtained, where $C_{1}$ joins $P$ and $P_{1}$ in $S_{1}$, and $C_{k}$ joins $P_{k-1}$ and $Q$ in $S_{k}$. The continuum $C=\sum_{1}^{k} C_{i}$ fulfills the condition stated in the theorem. $\dagger$

CoRollary. In $E_{n}(n>1)$ the complement of a zero-dimensional set is strongly connected.

THEOREM 5. In order that a set in $E_{2}$ should be zero-dimensional it is necessary and sufficient that it should be accessible from all sides.

That the condition is necessary follows from Theorem 2.

The condition is also sufficient. Let $P$ be any point of $M$. I shall show that $M$ is locally separated at $P$. If $\epsilon$ is any positive number, let $C_{1}, C_{2}, C_{3}$, and $C_{4}$ be circles with centers at $P$ with radii $\epsilon / 4, \epsilon / 2,3 \epsilon / 4$, and $\epsilon$, respectively. On a radius of $C_{4}$, let the intersections with the circles $C_{i}(i=1,2,3,4)$ occur in the order Plkji, and on the radius diametrically opposite let $a$ and $b$ be

*If "region" be replaced by "sphere," the definition of simple chain and Theorem 10 as given on pp. 134-135 of R. L. Moore's Foundations of plane analysis situs (these Transactions, vol. 17 (1916), pp. 131-164) will suffice for reference here.

$\dagger$ It was shown by Urysohn (loc. cit., vol. 8, p. 355) that if $M$ is an $F_{\sigma}$ of dimension $<n-1$ in $E_{n}(n>1)$ and $D$ is a connected domain, then $D-D \times M$ is strongly connected. (As a matter of fact, as I have pointed out in my paper Concerning a theorem of $J$. R. Kline, not yet published, $D-D \times M$ is arcwise connected.) Theorem $4 \mathrm{a}$ shows that for the case $n=2$ the restriction that $M$ be an $F_{\sigma}$ is unnecessary. 
the intersections with $C_{1}$ and $C_{2}$, respectively. On $C_{1}$ let $m, n, o$ be points in the order (counter-clockwise) $l m n o a$. Extend the radius $P n$ of $C_{1}$ to meet $C_{2}$ at $c$; and the radius $P m$ to meet $C_{2}$ and $C_{3}$ at $d$ and $q$, respectively. On $C_{2}$ let $f$ and $e$ be selected so that the order (counter-clockwise) $k f e d c b$ is obtained. Let a radius of $C_{4}$ through $f$ cut $C_{3}$ and $C_{4}$ in $g$ and $h$, respectively, and a radius through $e$ meet $C_{3}$ in $s$. Denoting straight line intervals by brackets, and arcs of circles by parentheses, define simple closed curves $J_{1}$ and $J_{2}$ as follows:

$$
J_{1}=[P a b]+(b c d e f)+[f g h]+(i h)+[i j k l]+(l m n o)+[o P],
$$

(ih) being so chosen that $J_{1}$ encloses points of $[n c]$;

$$
J_{2}=[P m d q]+(q j g s)+[s e]+(e f k b c)+[c n P] .
$$

That arc $j g$ on $C_{3}$ which does not contain $s$ forms, with the portion $[j i]+(i h)+[h g]$ of $J_{1}$, a simple closed curve $J_{3}$. Let $Q_{1}$ be a point interior to $J_{3}$, not belonging to $M$. As $Q_{1}$ is interior to $J_{1}$, there exists a continuum $K_{1}$ containing $Q_{1}$ and $P$, and lying, except for $P$, wholly interior to $J_{1}$, and containing, except for $P$, only points of $E_{2}-M$. Let

$$
J_{4}=[f g]+(g s)+[s e]+(e f),
$$

where ( $g s)$ does not contain $j$, and (ef) does not contain $k$, and let $Q_{2}$ be a point of $E_{2}-M$ interior to $J_{4}$. As $Q_{2}$ is also interior to $J_{2}$, there exists a continuum $K_{2}$ containing $Q_{2}$ and $P$, lying, except for $P$, wholly interior to $J_{2}$, and containing, except for $P$, only points of $E_{2}-M$.

The continuum $K_{1}$ contains a continuum $T_{1}$ which lies wholly within or on the boundary of the annular domain bounded by $C_{1}$ and $C_{3}$, and contains points on both $C_{1}$ and $C_{3}{ }^{*}$ The points of $T_{1}$ on $C_{3}$ lie on the arc $j g$ of $J_{3}$, and the points of $T_{1}$ on $C_{1}$ are on that arc ao of $C_{1}$ which does not contain $n$. The continuum $K_{2}$ contains a continuum $T_{2}$ which lies wholly within or on the simple closed curve $J_{5}$ defined as follows:

$$
J_{5}=[f g]+(g j q)+[q d m]+(m n)+[n c]+(c b k f),
$$

where $(m n)$ does not contain $a$, and such that $T_{2}$ has points on both $[f g]$ and $(m n)$.

The continuum $T=T_{1}+T_{2}$ does not contain $P$ and hence is a subset of $E_{2}-M$. If $D$ is that complementary domain of $T$ determined by $P$, then $D$ is a region whose boundary is a subset of $T$, and such that $D$ contains no point on or exterior to $C_{4}$. The diameter of $D$ is less than $\epsilon$, and hence $M$ is locally separated at $P$.

* Cf. Anna M. Mullikin, Certain theorems relating to plane connected point sets, these Transactions, vol. 24 (1922), pp. 144-162, Theorem 1. 
If a set $M$ in $E_{2}$ is accessible from all sides, then by Theorem 5 it is zerodimensional; and hence, by Theorem 2, $E_{2}$ is accessible from all sides with respect to $M$ and, by Theorem $3, E_{2}-M$ is strongly connected im kleinen. Hence the following corollary:

Corollary. If a set $M$ in $E_{2}$ is accessible from all sides, then $E_{2}$ is accessible from all sides with respect to $M$, and $E_{2}-M$ is strongly connected im kleinen.

Thus in $E_{2}$ accessibility from all sides of a set, and accessibility of $E_{2}$ from all sides with respect to that set, are equivalent properties.

That ordinary accessibility of points of $M$, in the sense that if $P$ is a point of $M$ and $Q$ a point of $E_{2}-M$ there exists a continuum $K$ containing $P$ and $Q$ and such that $K \times M=P$, is not sufficient, even where $M$ is punctiform and totally disconnected, to insure the zero-dimensionality of $M$ is shown by the example of a quasi-connected point set in my paper $A$ set which has no true quasi-components and which becomes connected upon the addition of a single point. ${ }^{*}$ The set described in this paper is accessible by arcs.

That in the definition of accessibility from all sides used above, arcs cannot be employed instead of unrestricted continua will be shown by the example given in $\$ 2$ of a zero-dimensional set which is not simply locally separated.

It is known $\dagger$ that the set, $I_{2}$, of all points in $E_{2}$ both of whose coördinates are irrational is homeomorphic with the set, $I_{1}$, of points in $E_{1}$ whose abscissas are irrational. Sierpinski has shown that zero-dimensional sets are homeomorphic with linear sets and hence with subsets of $I_{1}$. Hence every zerodimensional set is homeomorphic with a subset of $I_{2}$. This suggests the question: Is a zero-dimensional set in $E_{2}$ isotopic with a subset of $I_{2}$ ? It is to be noticed, first, that any subset of $I_{2}$ is simply locally separated as defined above, and that any set isotopic with a subset of $I_{2}$ must accordingly be simply locally separated. Hence I shall first show that the above question cannot be answered affirmatively by giving an example of a zero-dimensional set in $\vec{E}_{2}$ which is not simply locally separated at any point.

* Bulletin of the American Mathematical Society, vol. 33 (1927), pp. 423-427.

$\dagger$ Cf. M. Fréchet, Les dimensions d'un ensemble abstrait, Mathematische Annalen, vol. 68 (1910), pp. 145-168. See especially p. 154.

‡ Cf. Menger, Bericht über die Dimensionstheorie, loc. cit., pp. 125-126, and references given therein. 
Consider, first, a set $M$ constructed as follows: Let $T$ be a unit square, and for each positive integer $n$ divide $T$ into $n^{2}$ equal squares, letting $T_{n}$ denote the corresponding set of $n^{2}$ squares. For each square, $t$, of $T_{n}$, construct a continuum $K(t)$ of which every subcontinuum is indecomposable* and which lies wholly within $t$ except that it contains four points on $t$, these being the mid-points of the four sides of $t$. For each $n$ let $M_{n}$ denote the set of points obtained by adding together the point sets $K(t)$ for all squares $t$ of $T_{n}$. Let $M=\sum_{1}^{\infty} M_{i}$. This set, $M$, was first constructed by R. L. Moore $\dagger$ as an example of a connected and connected im kleinen point set which contains no arc. The point set which I wish now to consider is the complement of $M$ within and on $T$. Denote this set by $N$.

The point set $N$ is a zero-dimensional $G_{\delta} \ddagger$ and is everywhere dense in $T$ and its interior. For if $P$ is a point of $N$ within $T$ and $C$ is a circle with center at $P$, there exists a positive integer $n$ such that not only does a square $t$ of $T_{n}$, within or on which $P$ lies, lie wholly within $C$, but also all those squares of $T_{n}$ adjacent to $t$. The eight continua $K_{t}$ constructed relative to $T_{n}$ in these adjacent squares form a continuum $F$. That complementary domain $D$ of $F$ which contains $P$ is a region which lies wholly interior to $C$ and whose boundary contains no point of $N$, being a subset of $F$ and hence of $M$. Then $N$ is locally separated at $P$. In case $P$ is on $T$ it can be shown in a similar way that $N$ is locally separated at $P$. That $N$ is everywhere dense in $T$ and its interior is evident since every arc interior to $T$ contains points of $N$. That $N$ is a $G_{\delta}$ is evident since $M$ is an $F_{\sigma}$. But it is obvious that $N$ is not simply locally separated at any point and consequently $N$ is not isotopic with $I_{2}$.

By an extension of this example there can be obtained an example of a zero-dimensional set which is dense everywhere in $E_{2}$, but which is not isotopic with $I_{2}$. This comment is of interest, perhaps, in that it relates to the analogue, for punctiform uncountable sets, of a theorem first given by Fréchet $\S$ and later by Urysohn $\|$ to the effect that all denumerable sets dense in $E_{n}$ are isotopic. If there exists any analogue of this theorem for uncountable sets, the example just indicated shows that zero-dimensionality is not a sufficient condition for isotopism of such sets.

* Cf. B. Knaster, Un continu dont tout sous-continu est indécomposable, Fundamenta Mathematicae, vol. 3 (1922), pp. 247-286.

$\dagger$ R. L. Moore, $A$ connected and regular point set which contains no arc, Bulletin of the American Mathematical Society, vol. 32 (1926), pp. 331-332.

$\ddagger A G_{8}$ is a set of points common to a denumerable infinity of open sets.

$\S$ M. Fréchet, loc. cit., p. 159.

II P. Urysohn, Sur les multiplicités Cantoriennes, loc. cit., vol. 7, pp. $83 \mathrm{ff}$. Urysohn states that Fréchet's proof seems to him insufficient. 
I shall now proceed to establish a condition which characterizes those sets which are isotopic with subsets of $I_{2}$.

Theorem 6. Let $M$ be a point set in $E_{2}$. Then in order that $M$ should be simply locally separated, it is necessary and sufficient that $E_{2}$ be arcwise accessible from all sides with respect to $M$.

The condition is necessary. Let $M$ be a simple locally separated set in $E_{2}$, and let $P$ be any point of $E_{2}$. Let $J$ be any simple closed curve containing $P$. Let $D$ be the bounded domain complementary to $J$, and let $Q$ be any point of $D$.

There exists a simple closed curve $K$ which contains $P$ and $Q$, and which lies, except for $P$, wholly in $D$. Denote the interior of $K$ by $R$. There exists an $\operatorname{arc} t$ which has $P$ and $Q$ as end points, and which lies, except for these two points, entirely in $R$.

Denote the point set $M \times[K-(P+Q)]$ by $M_{1}$.

Since $M$ is simply locally separated, there exists, by Theorem 1a, a sequence, $G$, of Jordan regions, which covers $M$ in the Vitali sense and has property $\mathrm{H}$, and such that the boundaries of the regions of $G$ contain no points of $M$.

If $x$ is a point of $M_{1}$, there exists a region $g_{x}$ of the collection $G$ which contains $x$ and such that $g_{x}^{\prime}$ lies wholly in $D$ and contains no point of $t$. The set of all points which lie in regions of the type $g_{x}$ together with their boundaries denote by $T$. The set of points $T+K$ is a continuum $C$. Furthermore, $C$ is a continuous curve. To show this, I shall employ Sierpinski's characterization* of a continuous curve; i.e., a bounded continuum $N$ is a continuous curve provided that for every positive number $e, N$ is the sum of a finite collection of continua each of which is of diameter less than $e$.

Let $e$ be any positive number. Then $K$, being a continuous curve, is the sum of a finite collection of continua, $C_{1}, C_{2}, \cdots, C_{n}$, each of which is of diameter less than $e / 4$. Since $G$ covers $M$ in the Vitali sense, there is only a finite number of regions of $G$ of diameter $>e / 4$. Hence those regions of $G$ which constitute part of $T$ and are of diameter $>e / 4$ are finite in number, and as a Jordan region together with its boundary forms a continuous curve, each of them together with its boundary is the sum of a finite number of continua of diameters less than $e$; denote the set of all such continua by $G_{1}$. If $g_{x}$ is a region which constitutes part of $T$ and is of diameter less than $e / 4$, and if $C_{i}(1 \leqq i \leqq n)$ is a part of $K$ which contains a point of $g_{x}^{\prime}$, then $C_{i}$,

*W. Sierpinski, Sur une condition pour qu'un continu soit une courbe jordanienne, Fundamenta Mathematicae, vol. 1 (1920), pp. 44-60. 
together with all such regions $g_{x}$ and their boundaries forms a continuum $C_{i}^{\prime}$. That $C_{i}^{\prime}$ is of diameter less than $e$ is obvious. Therefore $C$ is the sum of a finite collection of continua, viz., $C_{1}^{\prime}, C_{2}^{\prime}, \cdots, C_{n}^{\prime}$, and the continua of the collection $G_{1}$, all of which are of diameter less than $e$, and hence is a continuous curve.

If $z$ is an interior point of $t$, denote by $D_{z}$ that complementary domain of $C$ determined by $z$. Denote the outer boundary of $D_{z}$ by $B$. By a theorem due to R. L. Moore, ${ }^{*} B$ is a simple closed curve. That $B$ contains $P$ and $Q$ is easily seen, and clearly $B$ lies wholly in $D$, except for $P$, and contains no point of $M$ except possibly $P$ and $Q$. As $B$ is the sum of two arcs whose end points are $P$ and $Q$, the condition stated in the theorem is proved necessary.

To show that the condition stated in the theorem is sufficient a modification of the proof of Theorem 5 may be employed.

TheOREM 6a. In order that a point set in $E_{2}$ should be simply locally separated it is necessary and sufficient that it be arcwise accessible from all sides.

CoRollary. If a set $M$ in $E_{2}$ is arcwise accessible from all sides, then $E_{2}$ is arcwise accessible from all sides with respect to $M$, and $E_{2}-M$ is arcwise connected im kleinen; and if $P$ and $Q$ are points of a connected domain $D$ in $E_{2}$ there is an arc from $P$ to $Q$ which lies wholly in $D$ and contains no point of $M$ except possibly $P$ and $Q$.

The proof of the latter part of this corollary can be obtained by use of the simple chain idea (see proof of Theorem $4 \mathrm{a}$ ), the arcwise accessibility of $E_{2}$ with respect to $M$, and of the fact that if $C$ is a circle enclosing two points $A$ and $B$ there exists a simple closed curve within $C$ which contains $A$ and encloses $B$.

Theorem 7. In $E_{2}$, let $M$ be a point set and let $I_{2}$ denote the set of all points both of whose coördinates are irrational. Then in order that $M$ should be isotopic with a subset of $I_{2}$, it is necessary and sufficient that it be simply locally separated.

That the condition stated in the theorem is necessary is obvious, since $I_{2}$ is itself simply locally separated.

To prove the condition sufficient, I shall proceed by methods based on R. L. Moore's Concerning a set of postulates for plane analysis situs. $\dagger$ I shall therefore first state a series of lemmas which are adaptations, as signified

\footnotetext{
* Concerning continuous curves in the plane, loc. cit., Theorem 4. That the boundary of $D_{\text {s }}$ is itself a continuous curve is demonstrated by Moore in the proof of Theorem 4.

† These Transactions, vol. 20 (1919), pp. 169-178.
} 
in each case, of Moore's theorems B-G. It will be understood that when a theorem is designated by letter it is one of Moore's theorems, and that when a theorem is designated by number it refers to a theorem in the present paper. Wherever a proof is not indicated it will be understood that the proof is only a slight modification of Moore's proof with the use of Theorem 6 .

Lemma 3 (Adaptation of Theorem B). If $J$ and $L$ are two simple closed curves and $A$ and $B$ are two distinct points of $J \times\left(E_{2}-M\right)$ each of which is either not on $L$ at all or on some segment that is common to $J$ and $L$, then there exists an arc from $A$ to $B$ which lies entirely in $E_{2}-M$ and, except for its end points, within $J$, and has not more than a finite number of points in common with L.

The proof of Lemma 3 is similar to that of Theorem B, except that use is made of Theorem 6 (a) in establishing the existence of the arc $A B$ in $E_{2}-M$, in the first sentence of the proof as given by Moore, and (b) in establishing the existence of the $\operatorname{arcs} A_{n} Z_{n} B_{n}$ of lines 10-12, page 172 , so that these have, in addition to the properties outlined there, the further property that they lie in $E_{2}-M$.

LEMma 4 (Adaptation of Theorem C). If the closed curve $g$ lies in $E_{2}-M$ and has only a finite number of points in common with the closed curve $A B C D A$ and does not contain $A, B, C$, or $D$, then the interior of $A B C D A$ can be divided by double ruling* such that (1) the arcs of this ruling lie in $E_{2}-M,(2)$ the arcs of one of its single rulings are parallel to $A B$ and $C D$ and those of the other are parallel to $A D$ and $B C$, and (3) the subdivisions of $A B C D A$ made by this ruling are such that the interior of each one of them is either wholly within or wholly without $g$.

Lemma 5 (Adaptation of Theorem D). If $A B C D A$ is a simple closed curve and $G$ is a set of simple closed curves which lie wholly in $E_{2}-M$ and each point on or within $A B C D A$ is within some curve of the set $G$, then the interior of $A B C D A$ can be divided by a double ruling such that (1) the arcs of this ruling lie wholly in $E_{2}-M,(2)$ the arcs of one of its single rulings are parallel to $A B$ and $C D$ and those of the other are parallel to $A D$ and $B C$, and (3) the subdivisions of the interior of $A B C D A$ formed by these rulings are such that each lies within some curve of the set $G$.

Lemma 6 (Adaptation of Theorem E). If ABCDA is a simple closed curve there exist two sets of arcs, $\alpha_{1}$ and $\alpha_{2}$, such that (1) each arc of $\alpha_{1}$ lies wholly within $A B C D A$ except that its end points are on $A B$ and $C D,(2)$ each arc of

\footnotetext{
* For definitions see Moore's paper, loc. cit.
} 
$\alpha_{2}$ lies wholly within $A B C D A$ except that its end points are on $B C$ and $D A$, (3) $\alpha_{i}(i=1,2)$ is the sum of two collections of arcs, $a_{i}$ and $b_{i}$, such that (i) if $d$ is an arc of $b_{i}$, then $d$ is the sequential limiting set of a sequence of arcs of the set $a_{i}$ and $a_{i}$ is a denumerable set, (ii) the arcs of $a_{i}$ contain no points of $M,(4)$ each point on $A B C D A$, with the exception of $A, B, C$, and $D$, is an end point of either just one arc of $\alpha_{1}$ or just one arc of $\alpha_{2}$, (5) through each point within $A B C D A$ there is just one arc of $\alpha_{1}$ and just one arc of $\alpha_{2}$, (6) each arc of $\alpha_{1}$ has just one point in common with each arc of $\alpha_{2}$.

The proof of Lemma 6 is the same as that of Theorem $\mathrm{E}$, the curves in each set $\beta_{n}$ being selected in $E_{2}-M$, however.

LEMma 7 (Adaptation of Theorem F). There exists a denumerably infinite sequence of simple closed curves $J_{1}, J_{2}, J_{3}, \cdots$ such that every point of $E_{2}$ lies within at least one of them and such that for every $n, J_{n+1}$ encloses $J_{n}$, and $J_{n}$ lies wholly in $E_{2}-M$.

The proof of Lemma 7 is either a modification of the proof of Theorem $F$ or an application of Theorem 6 to a series of annular domains.

Lemma 8 (Adaptation of Theorem G). There exist in $E_{2}$ two sets, $G_{1}$ and $G_{2}$, of open curves such that (1) through each point there is just one curve of $G_{1}$ and just one curve of $G_{2},(2)$ each curve of $G_{1}$ has just one point in common with each curve of $G_{2},(3) G_{i}(i=1,2)$ consists of two sets of open curves $G_{i 1}$ and $G_{i 2}$ such that (a) no curve of the set $G_{i 1}$ contains a point of $M$, (b) the set $G_{i 1}$ is denumerable, (c) every curve of the set $G_{i 2}$ is the sequential limiting set of a sequence of curves of the set $G_{i 1}$.

To establish the sufficiency of the condition stated in Theorem 7, proceed as follows on the basis of Lemma 8: Let $g_{1}$ and $g_{2}$ be particular open curves of the collection $G_{11}$ and $G_{21}$, respectively. Between the points of $g_{1}$ and the points of the $x$-axis there is a one-to-one continuous correspondence in which the intersections of $g_{1}$ with curves of the set $G_{21}$ correspond to points on the $x$-axis whose abscissas are rational and the intersections of $g_{1}$ with curves of the set $G_{22}$ correspond to points on the $x$-axis whose abscissas are irrational, and in which the intersection of $g_{1}$ with $g_{2}$ corresponds to the point of the $x$ axis whose abscissa is zero. Similarly, between the points of $g_{2}$ and the points of the $y$-axis there exists a one-to-one continuous correspondence in which the intersections of $g_{2}$ with the curves of the collection $G_{11}$ correspond to the points of the $y$-axis whose ordinates are rational and the intersections with the curves of the collection $G_{12}$ correspond to the points whose ordinates are irrational and in which the intersection of $g_{1}$ and $g_{2}$ corresponds to the point whose ordinate is zero. 
Let $\Sigma_{1}$ denote the ordinary cartesian system of coördinates, and let $\Sigma_{2}$ denote a system of coördinates defined as follows: If $P$ is any point of $E_{2}$, the intersection of that curve of the collection $G_{2}$ which contains $P$ with $g_{1}$ corresponds, in the correspondence outlined above, to a point of the $x$-axis whose abscissa is, say, $x_{2}$; and the intersection of that curve of $G_{1}$ which contains $P$ with $g_{2}$ corresponds to a point of the $y$-axis whose ordinate is $y_{2}$; then the coördinates of $P$ in the system $\Sigma_{2}$ will be $\left(x_{2}, y_{2}\right)$. Then there exists a one-to-one continuous transformation of $E_{2}$ into itself in which two points correspond if and only if their respective coördinates in the two systems $\Sigma_{1}$ and $\Sigma_{2}$ are identical, each to each. Since points of $M$ lie only on curves of the sets $G_{21}$ and $G_{22}$, it is evident that these points correspond to points both of whose coördinates are irrational in the system $\Sigma_{1}$; i.e., the points of $M$ correspond to a subset of $I_{2}$.

As a consequence of Theorems $6 \mathrm{a}$ and 7 we have

THEOREM 7a. In order that a set of points in $E_{2}$ should be isotopic with a subset of $I_{2}$, it is necessary and sufficient that it be arcwise accessible from all sides.

THEOREM 8. In $E_{2}$, let $M$ be a punctiform $F_{\sigma}$; then $E_{2}$ is arcwise accessible from all sides with respect to $M$.

In my paper Concerning a theorem of $J$. R. Kline, I have shown that if, in $E_{2}, D$ is any connected domain and $N$ is.a punctiform $F_{\sigma}$, then the set $D-D \times N$ is arcwise connected. Accordingly, if $J$ is any simple closed curve and $P$ is a point of $J$, and $Q$ is a point of the region, $R$, bounded by $J$, it is easy to prove that there exists an $\operatorname{arc}$ from $P$ to $Q$ which lies, except for $P$, and possibly $Q$, wholly in the set $D \times\left(E_{2}-M\right)$.

As a consequence of Theorems 6 and 8 we have

THEOREM 9. In $E_{2}$ every punctiform $F_{\sigma}$ is simply locally separated.

As a consequence of Theorems 7 and 9 we have

THEOREM 10. In $E_{2}$ every punctiform $F_{\sigma}$ is isotopic with some subset of $I_{2}$, the set of all points both of whose coördinates are irrational.

\section{SOME PROBLEMS}

1. Under what conditions is a zero-dimensional set in $E_{n}(n>2)$ isotopic with a subset of $I_{n}$, the set of points all of whose coördinates are irrational?

* A set is an $F_{\sigma}$ if it is the sum of a denumerable collection of closed sets. Cf. F. Hausdorff, Grundzüge der Mengenlehre, Leipzig, 1914, pp. $304 \mathrm{ff}$. 
2. If $M$ is a punctiform $F_{\sigma}$ in $E_{n}(n>2)$, is $M$ isotopic with some subset of $I_{n}$ ?

3. When is a zero-dimensional set $M$ in $E_{n}(n>2)$ arcwise accessible from all sides, and when is $E_{n}$ arcwise accessible from all sides with respect to $M$ ? Are the two properties equivalent?

4. If $M$ is a zero-dimensional set in $E_{n}(n>2)$ under what conditions is the complement of $M$ arcwise connected? More generally, if $D$ is a connected domain, when is $D-D \times M$ arcwise connected; when are any two points of $D$, say $P$ and $Q$, the end points of an $\operatorname{arc}$ of $D$ which contains no point of $M$, except possibly $P$ and $Q$ ?

Problems 3 and 4 are obviously closely related, for it is easy to show that if any set $M$ (zero-dimensional or not) is such that $E_{n}$ is arcwise accessible from all sides with respect to $M$, and $D$ is a connected domain and $P$ and $Q$ are points of $D$, then $D$ contains an $\operatorname{arc}$ from $P$ to $Q$ which contains no point of $M$ except possibly $P$ and $Q$.

5. What is the maximum dimension of a point set which is accessible from all sides in $E_{n}$ ?

The solution of Problem 5 for $n=2$ is obviously given by Theorem 5 .

6. Let $M$ be an arbitrary point set and $D$ a connected domain in $E_{n}$. Is there a more general condition than that given by Urysohn (see footnote accompanying Theorem $4 \mathrm{a}$ ) sufficient to ensure the strong connectivity of $D-D \times M$ ?

University of Michigan, ANN ARbor, Mich. 